Document downloaded from:

http://hdl.handle.net/10251/99608

This paper must be cited as:

Díaz Calleja, R.; Sanchis Sánchez, MJ.; Alvarez, C.; Riande, E. (1997). Relaxation behavior of semiflexible polymers at very low frequencies. Journal of Applied Physics. 81(8):36853691. doi:10.1063/1.364743

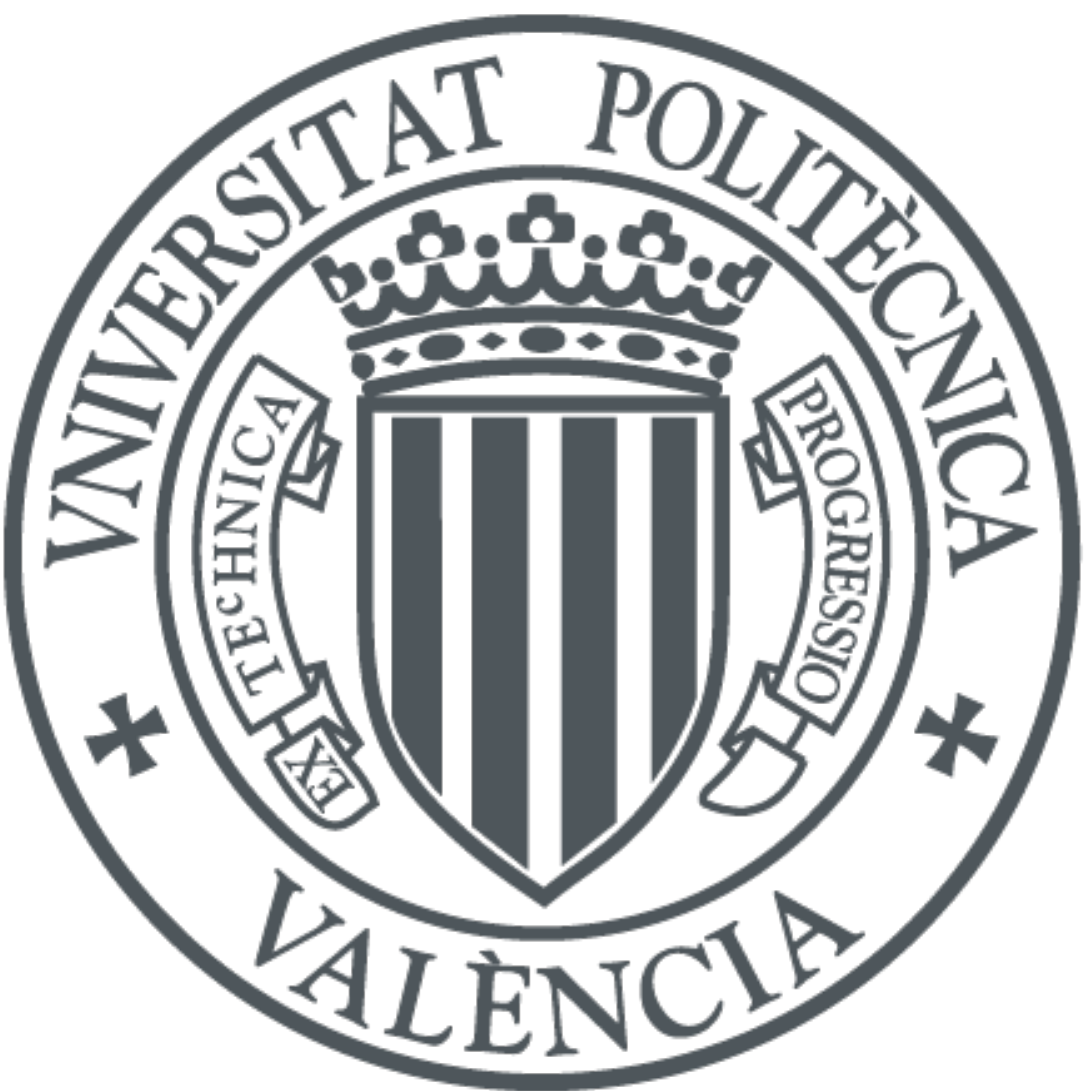

The final publication is available at

https://doi.org/10.1063/1.364743

Copyright American Institute of Physics

Additional Information 


\title{
Relaxation behavior of semiflexible polymers at very low frequencies
}

\author{
Ricardo Díaz-Calleja and M. J. Sanchis \\ Departamento de Termodinámica Aplicada, ETSII, UPV, 46071 Valencia, Spain
}

Cristina Alvarez and Evaristo Riande

Instituto de Ciencia y Tecnología de Polímeros (CSIC), 28006 Madrid, Spain

The dielectric activity of poly(monocyclohexylmethylene itaconate) ( PMCI) and poly(dicyclohexylmethylene itaconate) ( PDCI) in the glassy region and in the glass-rubber transition is studied by thermostimulated discharge current (TSDC) techniques. The spectra obtained by global TSDC experiments show a prominent $\alpha$-glass-rubber relaxation peak with maxima located at 97 and $55^{\circ} \mathrm{C}$ for PMCI and PDCI, respectively, following in decreasing order of temperature for a well developed $\beta$ absorption and a comparatively low intensity relaxation. Better resolution of the relaxation behavior of these polymers in the glassy region is obtained by calculating the components of the complex dielectric permittivity $\epsilon^{*}$ at extremely low frequencies from partial TSDC experiments. The ac spectra thus obtained suggest that the $\beta$ absorption is composed by two relaxations, each of them presumably associated with the motions of a side group. The differences observed in these spectra with those reported for the $\beta$ mechanical relaxation reported for these polymers in the literature are interpreted in terms of the restrictions that the side groups produce in the conformational space of phase of the backbone. These restrictions also explain the small changes in enthalpy in the glass-rubber transition which preclude the possibility of obtaining the glass-rubber transition temperature of these polymers by differential scanning calorimetric techniques.

\section{INTRODUCTION}

The response of polymers in the liquid state to the perturbation of a dynamic force field involves molecular motions whose complexity increases as the frequency of the perturbative field decreases. If the response reflecting a loss property of the system is plotted in the frequency domain, an absorption or $\beta$ relaxation is detected at high frequencies caused by local conformational transitions, followed in the order of decreasing frequency by a prominent $\alpha$ relaxation process arising from generalized microbrownian motions. ${ }^{1}$ At very low frequencies, the mechanical relaxation spectra of polymers exhibit a third relaxation peak produced by motions involving the wholly molecular chains. ${ }^{2}$ This absorption, called the normal mode relaxation process, is also detected in the dielectric relaxation spectra of polymers in which the dipoles associated with bonds or groups of bonds have a component parallel and the other perpendicular to the chain contour. ${ }^{3-5}$ Whereas the location of both the $\alpha$ and $\beta$ relaxations in the frequency domain are nearly independent of the chains' length, the frequency associated with the normal mode peak scales with the -1 and -3.4 power of molecular weight for unentangled and entangled polymer chains, respectively. By plotting the loss at a determined frequency against temperature, the $\beta, \alpha$ and normal relaxation processes appear in the order of increasing temperature. The $\beta$ and $\alpha$ processes are associated with molecular motions in the glassy state and the glass-rubber transition, respectively. The normal mode process is centered at a temperature $T_{n}\left(>T_{g}\right)$ whose value diminishes as the frequency increases.
The dielectric $\beta$ relaxation is extremely broad in the frequency domain suggesting a variety of local environments, each of them with its own barrier system. ${ }^{6,7}$ Although this process has currently been attributed to molecular motions preferentially taking place in the side groups, ${ }^{1,8-10}$ secondary absorptions are also detected in the relaxation spectra of molecular chains without flexible side groups in their structure. ${ }^{1}$ Computer simulations suggest that secondary processes in these latter chains arise from conformational transitions occurring in pairs. ${ }^{11,12}$ The two transitions take place nearby in space and time, so that the ends of the chains are not required to move. As a consequence, the relaxation times associated with secondary processes are independent on molecular weight. Molecular cooperativity is also invoked in the microbrownian molecular motions which produce the $\alpha$ glassrubber process, as the independence of this process on molecular weight for long chains suggests.

In order to get a better understanding of the molecular origin of the secondary relaxations, it is advisable to carry out relaxation studies on amorphous polymers in which the conformational space of phase is severely reduced. Interesting candidates for this task are monoesters and diesters of poly(itaconic acid) such as poly(monocyclohexylmethylene itaconate) (PMCI) and poly(dicyclohexylmethylene itaconate) (PDCI), respectively, whose structural units are schematically represented in Fig. 1. A close inspection of the structure of these polymers suggests that the flexible side groups must hinder the molecular motions about the main chain. ${ }^{13,14}$ The mechanical spectra of both PMCI and PDCI, expressed in terms of the loss relaxation modulus, present an $\alpha$-glass-rubber peak whose intensity is comparable or lower than the intensities of the two secondary peaks, $\beta$ and $\gamma$, detected in the glassy region. In fact, the intensity of the 


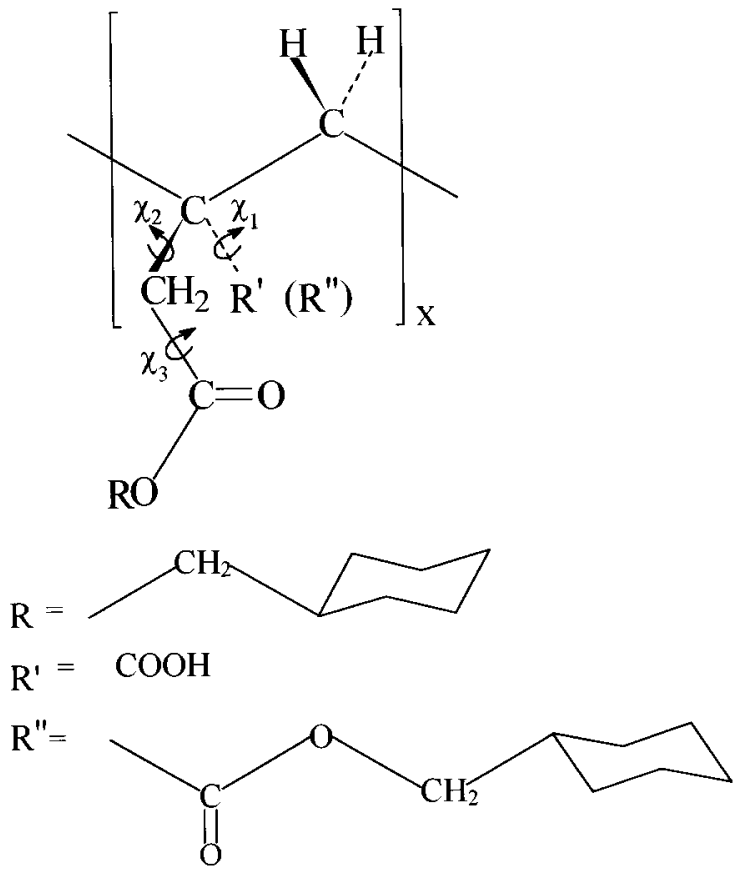

Figure 1. Sketch of the repeating units of PDCI and PMCI. The substituents in PDCI and PMCI are, respectively, $R, R^{\prime \prime}$, and $R, R^{\prime}$.

peaks for PDCI decreases in the order $\beta>\gamma>\alpha$. Three absorptions $(\alpha, \beta$, and $\gamma)$ also exhibit the dielectric relaxation spectra of these polymers, but in this case their intensities increase in the order $\gamma<\beta<\alpha$. The high dielectric and mechanical activity exhibited by both PMCI and PDCI in the glassy state prompted us to study their dielectric responses from thermostimulated discharge current (TSDC) experiments with the aim of obtaining a more detailed information on the dielectric subglass absorptions of these polymers at extremely low frequencies.

\section{EXPERIMENT}

Both poly(monocyclohexylmethylene itaconate) (PMCI) and poly(dicyclohexylmethylene itaconate) (PDCI) were obtained by radical polymerization of monocyclohexylmethylene itaconate and dicyclohexylmethylene itaconate, respectively, at $70^{\circ} \mathrm{C}$, using $\alpha, \alpha^{\prime}$-azobis(isobutyronitrile) (AIBN) as initiator. ${ }^{15}$ The polymers were dissolved several times in tetrahydrofurane, reprecipitated with diethyl ether, and finally dried in vacuo at $100{ }^{\circ} \mathrm{C}$. The weight-average molecular weight of PMCI and PDCI were 154,000 and 170,000 $\mathrm{g} / \mathrm{mol}$, respectively. The weak change occurring in enthalpy in the glass-rubber transition of the esters of poly(itaconic acid) precluded the possibility of obtaining the calorimetric glass-transition temperature of PMCI.

Thermally stimulated discharge current curves were obtained with a TSC-RMA (Solomat) apparatus on polarized pills of the polymers of $0.2 \mathrm{~mm}$ thickness. The global dependence of the discharge intensity current was obtained by poling the pills at $75{ }^{\circ} \mathrm{C}$ for 10 min under a potential of 500 $\mathrm{V} / \mathrm{mm}$ and further quenching them at $-140{ }^{\circ} \mathrm{C}$. The electric field was further removed and the electrodes were shortcircuited and kept at the quenching temperature for $5 \mathrm{~min}$.

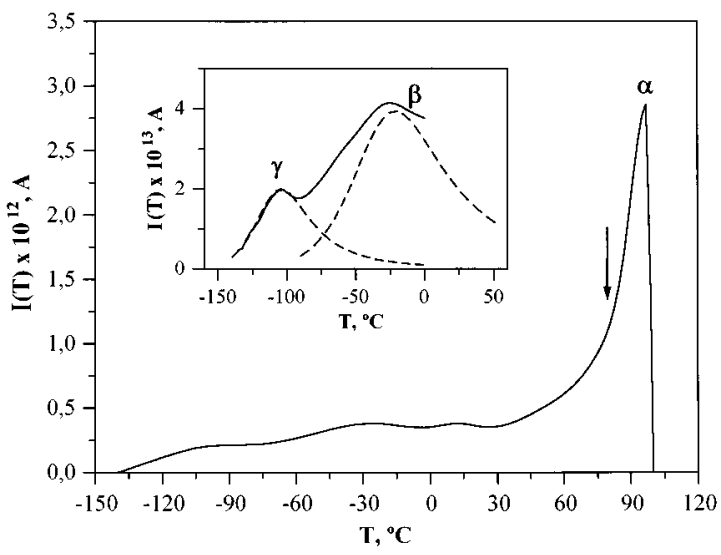

Figure 2. Global TSDC curve for PMCI with the arrow indicating the polar-ization temperature. Inset: details of the curve in the glassy region.

Then the electrode assembly was warmed at a constant heating rate of $7{ }^{\circ} \mathrm{C} / \mathrm{min}$. From the time derivative of the depolarization $(J=d P / d t)$, the global discharge current curve as function of temperature was obtained. Partial polarization discharge curves were also obtained at different temperatures in the glassy region, using poling windows of $10{ }^{\circ} \mathrm{C}$.

\section{RESULTS}

The curves showing the temperature dependence of the global depolarization of PMCI and PDCI are shown in Figs. 2 and 3, respectively. The TSDC curve for PMCI presents two broad peaks of relatively low intensity corresponding, in order of increasing temperature, to the $\gamma$ and $\beta$ relaxations. At higher temperatures, a prominent $\alpha$ absorption associated with the glass-rubber relaxation appears. The same curve for PDCI exhibits a rather narrow $\beta$ peak, in comparison with that of PMCI, whose intensity is much larger than that of the $\gamma$ absorption. By associating the glass-transition temperatures to the maximum of the $\alpha$ peaks, the values of $T_{g}$ for PMCI and PDCI are 55 and $97^{\circ} \mathrm{C}$, respectively.

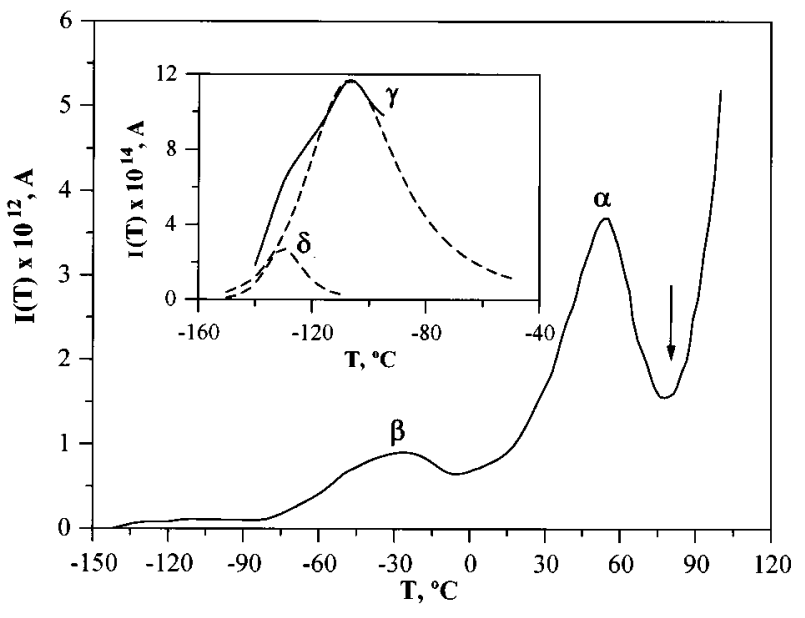

Figure 3. Global TSDC curve for PDCI with the arrow indicating the poling temperature. Inset: details of the curve in the glassy region. 
More detailed representations of the global depolarization curves in the glassy state, shown for both polymers in the insets of Figs. 2 and 3, indicate that the $\gamma$ peak for PMCI is narrower and better defined than the same peak for PDCI which, in turn, seems to be the result of two $(\delta$ and $\gamma)$ overlapping relaxations. The peak corresponding to each relaxation was resolved by fitting the absorption curves to the Fuoss-Kirkwood empirical expression ${ }^{16}$

$$
I=I_{\max }^{\prime \prime} \operatorname{sech}\left[m \frac{E_{a}}{R}\left(\bar{T}_{T} \frac{1}{T_{\max }}\right)\right]
$$

where $I_{\max }^{\prime \prime}$ is the intensity of the absorption at the peak maximum, $E_{a}$ is its activation energy, and $m$ is an empirical parameter related to the width of the peak in such a way that the broader the relaxation, the lower the value of $m$. In fact, $m$ lies in the range $0<m \leqslant 1$, reaching the value of the unity for a Debye-type peak. By plotting $\cosh ^{-1}\left(I_{\max }^{\prime \prime} / I\right)$ against $1 / T$ a straight line is obtained for symmetric peaks from whose slope the value of $m E_{a} / R$ can be obtained. The value of this quantity in conjunction with $T_{\max }$ allowed the deconvolution of the overlapping $\delta$ and $\gamma$ peaks (Fig. 3). From this analysis one finds that the $\delta$ peak is centered at $\sim-130^{\circ} \mathrm{C}$, nearly $25^{\circ} \mathrm{C}$ below the location of the $\gamma$ absorption which is centered at $\sim-105^{\circ} \mathrm{C}$ for both PDCI and PMCI. The $\beta$ absorption corresponding to PDCI is significantly narrower than that of PMCI, the intensity of this relaxation at the peak maximum for the former polymer being nearly two times that of the latter. In general, the sub-glass absorptions of these polymers are much better defined in the global TSDC curves than in the ac dielectric spectra obtained at frequencies above $1 \mathrm{~Hz}{ }^{13,14}$

The equivalent frequency at which ac experiments should be performed in order to obtain a loss peak having the maximal temperature as the global TSDC peak can be obtained from the current density $J(T)$, by means of the following expression: ${ }^{17,18}$

$$
\begin{aligned}
J(T)= & \frac{P_{e}\left(T_{p}\right)}{\tau_{0}} \exp \left(-\frac{E_{a}}{k T}\right) \\
& \times \exp \left[-\frac{1}{h \tau_{0}} \int_{T_{0}}^{T} \exp \left(-\frac{E_{a}}{k T^{\prime}}\right) d T^{\prime}\right],
\end{aligned}
$$

where $k$ is the Boltzmann constant, $P_{e}\left(T_{p}\right)$ is the steady state polarization at the polarization temperature $T_{p}$ and $h=d T / d t$ is the heating rate whose value, in the conditions the experiments were run, was $7{ }^{\circ} \mathrm{C} / \mathrm{min}$. The appearance of the preexponential factor $\tau_{0}$ and the activation energy $E_{a}$ in Eq. (2) is a consequence of assuming an Arrhenius-type dependence of the relaxation times on temperature. Since at the maximum temperature $d J(T) / d T=0$, the equivalent frequency, $f_{\text {eq }}$, can be obtained by differentiating Eq. (2), giving

$$
f_{\mathrm{eq}}=\frac{1}{2 \pi \tau\left(T_{m}\right)}=\frac{h E_{a}}{2 \pi k T_{m}^{2}}
$$

where $T_{m}$ is the temperature at the peak maximum and $E_{a}$ is the activation energy associated with the peak. The activation energy for the $\beta$ dielectric relaxation of PMCI is $\sim 15$ $\mathrm{kcal} \mathrm{mol}{ }^{-1}$ so that the value of the equivalent frequency lies

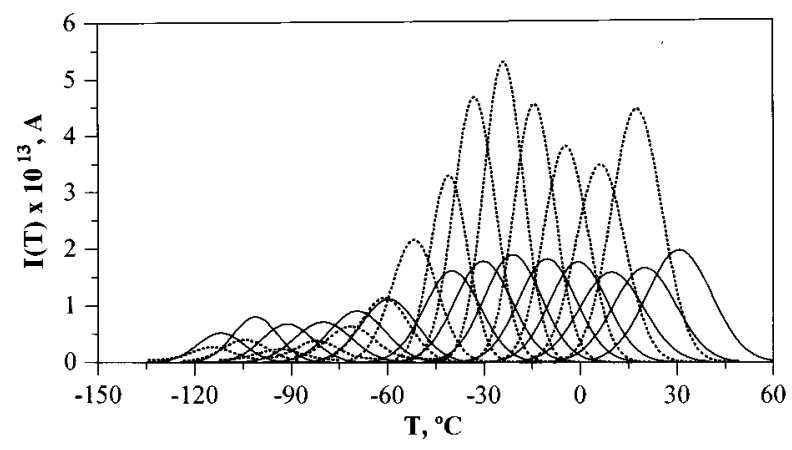

Figure 4. Elementary sub-glass TSDC spectra for PMCI (continuous line) and PDCI (discontinuous line). The lowest poling temperature is $-120^{\circ} \mathrm{C}$ and the interval of temperature between the poling temperatures of two consecu-tive peaks is $10^{\circ} \mathrm{C}$.

in the vicinity of $3 \times 10^{-3} \mathrm{~s}^{-1}$. A similar result is obtained for the equivalent frequency of the $\beta$ relaxation of PDCI.

With the aim of obtaining more detailed information on the sub-glass absorptions of itaconate polymers, elementary peaks in the glassy region were obtained by partial depolarization of poled samples. Partial depolarization curves for PDCI and PMCI, obtained at several poling temperatures, are shown in Fig. 4. A close inspection of these curves reveals two clearly differentiated regions corresponding to the $\gamma$ and $\beta$ relaxations. A zone of comparatively low dielectric activity lying in the range -120 to $-80{ }^{\circ} \mathrm{C}$ is followed by a region of high dielectric activity with a maximum located in the vicinity of $-25^{\circ} \mathrm{C}$, in consonance with the maximum obtained for the global TSDC spectra. It is worth noting that the intensity of the $\gamma$ relaxation for PMCI is somewhat larger than that corresponding to PDCI. The opposite occurs with the $\beta$ relaxation process. By assuming that each partial TSDC peak is described by a single relaxation time, its temperature dependence can be written as ${ }^{19}$

$$
\tau(T)=\frac{\int_{T_{i}}^{T} J(T) d T}{h J(t)}
$$

where $T_{i}$ is the initial depolarization temperature of each depolarization curve. The temperature dependence of the relaxation times follows Arrhenius behavior up to at least the half-width temperature of the peak, thus allowing the determination of the pre-exponential factors, $\tau_{0}$, and activation energies, $E_{a}$, associated with each peak. Whereas the values of the activation energy show a tendency to increase with the poling temperature, the values of $\tau_{0}$, though somewhat lower in the $\beta$ than in the $\gamma$ region, do not follow a definite trend. These results, represented in Fig. 5, suggest that the changes in activation energy with the poling temperature are significantly larger in the region of the $\beta$ relaxation than in the zone of the $\gamma$ absorption, as a consequence of the more complex motions which presumably occur in the $\beta$ relaxation process.

In general, the intensity of the elementary peaks undergoes a significant increase from the $\gamma$ to the $\beta$ region. The strength of each partial depolarization curve can quantitatively be expressed by ${ }^{20}$ 

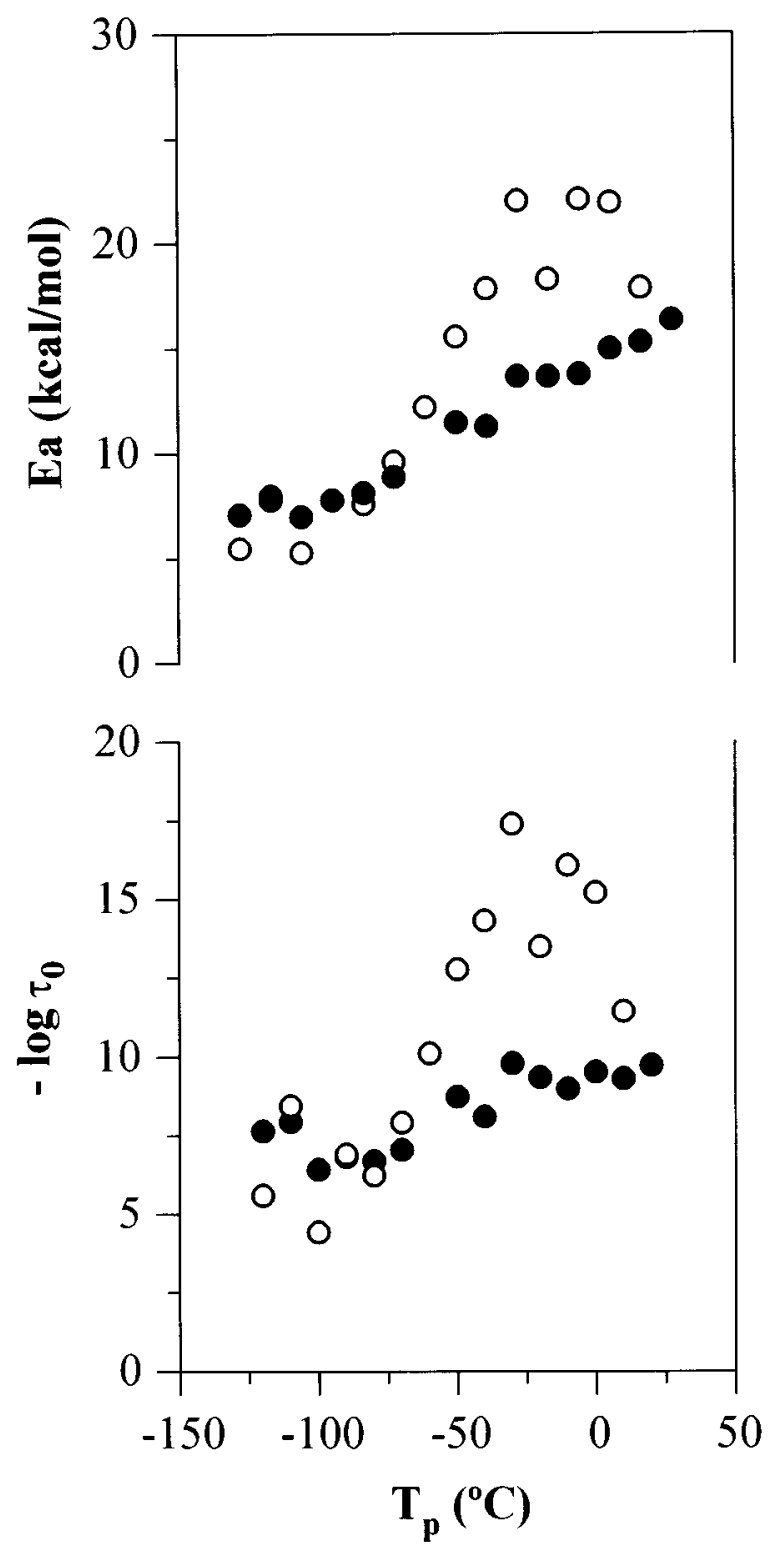

Figure 5. Evolution of the pre-exponential factor and the activation energy in the Arrhenius equation with the poling temperature for PMCI (filled circles) and PDCI (open circles).

$$
\Delta \epsilon_{g}=\frac{\int_{T_{0}}^{T_{\infty}} J(T) d T}{\epsilon_{0} h A E}
$$

where $\epsilon_{0}(=8.854 \mathrm{pF} / \mathrm{m})$ is the dielectric permittivity in vacuo, $A$ is the area of the pill, $E$ is the electric field, and $T_{0}$ and $T_{\infty}$ are, respectively, the extreme temperatures at the low and the high temperature sides of the peaks. The values of $\Delta \epsilon_{g}$ calculated by means of Eq. (5) are represented as a function of the poling temperature in Fig. 6. The curves obtained resemble those of the global depolarization curves. As expected, the relaxation strength of the depolarized curves in the $\gamma$ region is somewhat larger for PMCI, however the distribution of relaxation strengths associated with the peaks of $\mathrm{PDCI}$ in the $\beta$ region is rather narrow in comparison with that of PMCI.

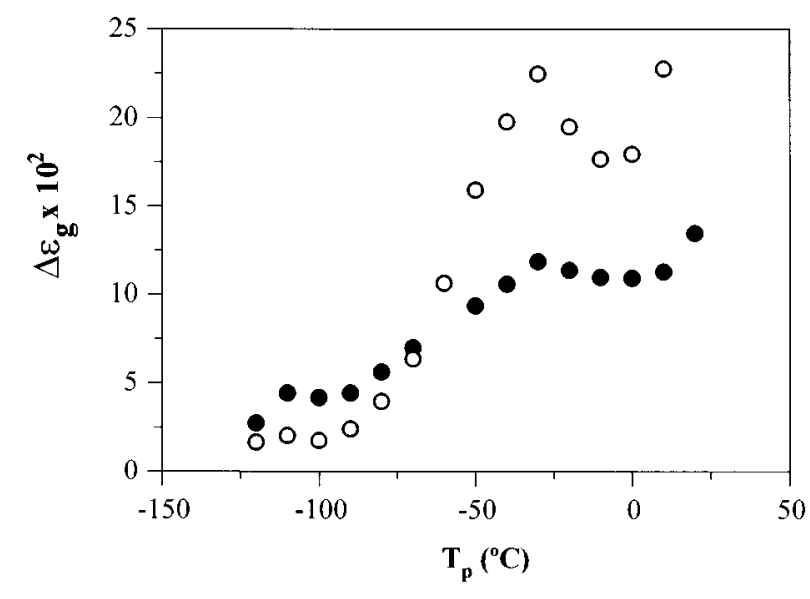

Figure 6. Dependence of the relaxation strength on the poling temperature for PMCI (filled circles) and PDCI (open circles).

A common characteristic of the ac loss curves is that the degree of overlapping of the responses of the different mechanisms increases with increasing frequency. At frequencies above $10 \mathrm{~Hz}$, the $\alpha$ absorption overlaps with the $\beta$ process and this with the $\gamma$ absorption precluding the possibility of obtaining a good picture of the dielectric activity occurring in these polymers in the glassy state. Consequently, values of both the real, $\epsilon^{\prime}$, and loss, $\epsilon^{\prime \prime}$, components of the complex dielectric permittivity, $\epsilon^{*}$, were tentatively obtained at extremely low frequencies from partial TSDC curves, by means of the following equations: $:^{20,21}$

$$
\begin{aligned}
& \epsilon^{\prime}(\omega)=\epsilon_{\infty}+\sum_{i=1}^{n} \frac{\left(\Delta \epsilon_{g}\right)_{i}}{1+\omega^{2} \tau_{i}^{2}(T)} \\
& \epsilon^{\prime \prime}(\omega)=\sum_{i=1}^{n} \frac{\left(\Delta \epsilon_{g}\right)_{i} \omega \tau_{i}(T)}{1+\omega^{2} \tau_{i}^{2}(T)}
\end{aligned}
$$

Values of $\epsilon^{\prime}$ in the frequency domain are represented at several temperatures in Fig. 7. In the same figure, values of $\epsilon^{\prime}$ are also plotted against temperature at several frequencies. It is important to note that the relaxation strength of the subglass dielectric process obtained from this analysis is 0.7 and 0.9 for PMCI and PDCI, respectively, in good agreement with the value of this quantity obtained from ac experiments. $^{13,14}$

The curves showing the temperature dependence of the dielectric loss exhibit a complicated pattern for PMCI. Taking the isochrone corresponding to $10^{-4} \mathrm{~Hz}$ as example, one can observe the presence of a broad $\beta$ peak covering an interval of temperature of $\sim 100{ }^{\circ} \mathrm{C}$. However, as the frequency of the isochrones decreases, the resolution of the broad peak increases in such a way that the $\beta$ relaxation seems to be the result of three overlapping processes. In Fig. 8 and for illustrative purposes, two of the three peaks obtained by deconvolution of the isochrone corresponding to $10^{-6} \mathrm{~Hz}$ are shown. It should be pointed out that the central peak does not fit to the Fuoss-Kirkwood equation.

The isochrones for PDCI, represented at several frequencies in Fig. 9, are somewhat different from those corresponding to PMCI. Thus the interval of temperature covered by the 

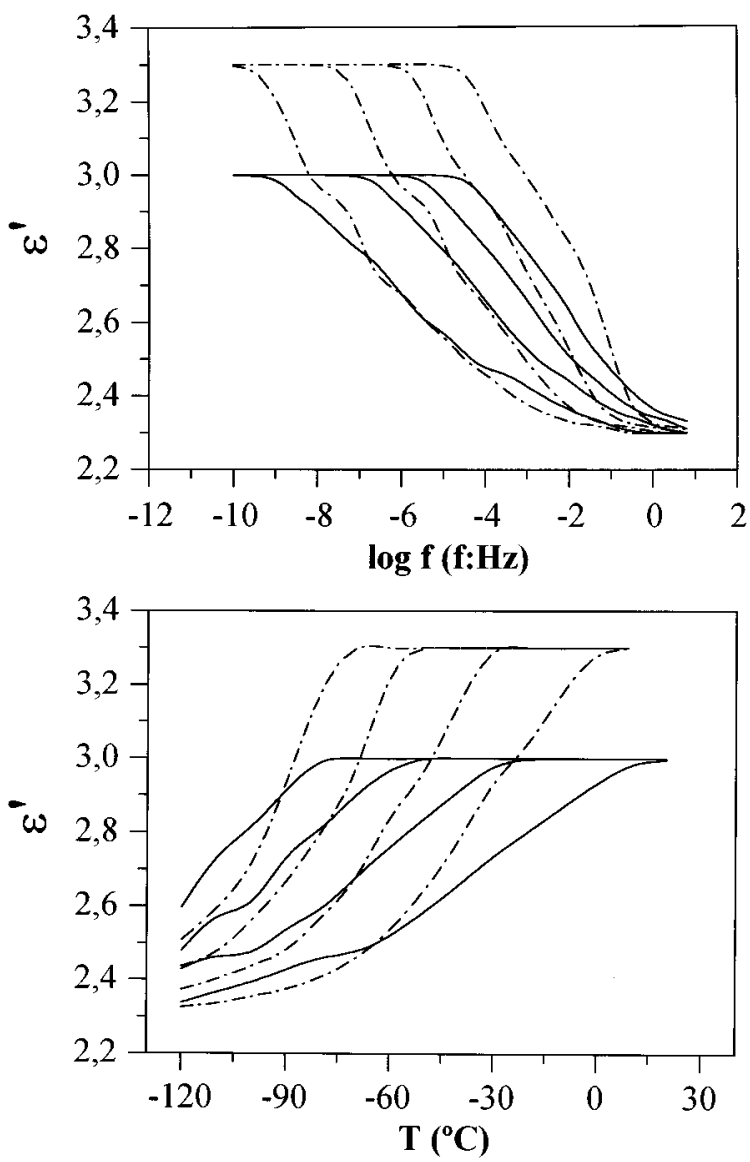

Figure 7. Top: Values of the real component of the complex permittivity, in the frequency domain, for PMCI (continuous curves) and PDCI (discontinuous curves) at the following temperatures: $-70,-50,-30$, and $-10{ }^{\circ} \mathrm{C}$. Bottom: Isochrones showing the temperature dependence of $\varepsilon^{\prime}$ on temperature for PMCI (continuous curves) and PDCI (discontinuous curves) at several frequencies: $10^{-10}, 10^{-8}, 10^{6}$, and $10^{-4} \mathrm{~Hz}$.

$\beta$ relaxation is considerably narrower for the former polymer than for the latter. Moreover, the temperature dependence of $\epsilon^{\prime \prime}$ for PMCI shows two well resolved peaks in the $\beta$ region at frequencies below $10^{-3} \mathrm{~Hz}$. At higher frequencies, the

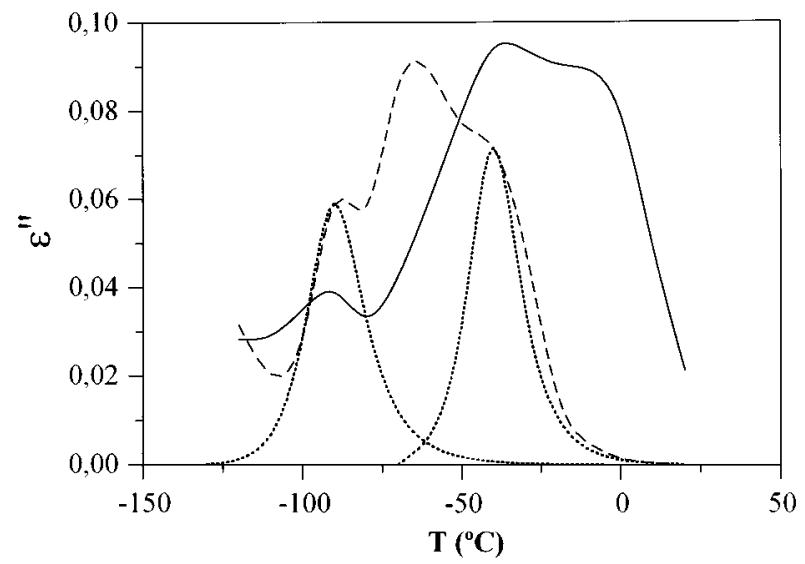

Figure 8. Temperature dependence of the dielectric loss for glassy PMCI at $10^{-4} \mathrm{~Hz}$ (continuous line) and $10^{-6} \mathrm{~Hz}$ (broken line). The peaks (....) flanking the central peak at $10^{-6} \mathrm{~Hz}$ are deconvoluted.

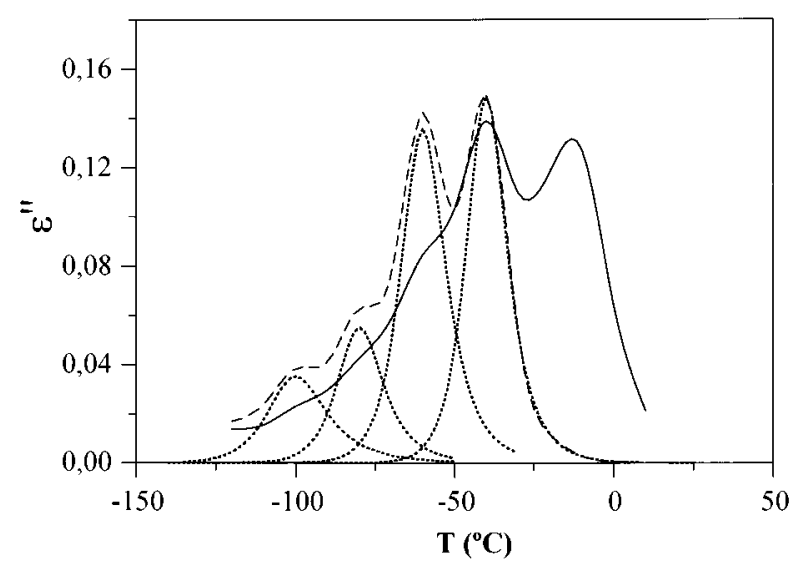

Figure. 9. Variation of the dielectric loss with temperature for glassy PDCI at $10^{-4} \mathrm{~Hz}$ (continuous line) and $10^{-6} \mathrm{~Hz}$ (broken line). The overlapping peaks at $10^{-6} \mathrm{~Hz}$ are deconvoluted.

absorptions in the $\beta$ region tend to overlap, forming a single peak.

Dielectric activity is also detected in the $\gamma$ region. Unfortunately, experimental difficulties precluded the possibility of obtaining partial stimulated depolarization curves at poling temperatures lying in the interval -150 to $-120{ }^{\circ} \mathrm{C}$ and, consequently, there is not sufficient experimental data to obtain the precise shape of the ac loss peaks in this zone.

\section{DISCUSSION}

Earlier studies carried out on the conformational statistics of PMCI and PDCI showed that a $2 \times 2$ rotational states scheme gives a good account of the effective dipole moment, $\mu_{\text {eff }}$, defined by

$$
\mu_{\mathrm{eff}}=\left(\frac{\left\langle\mu^{2}\right\rangle}{x}\right)^{1 / 2}
$$

where $\left\langle\mu^{2}\right\rangle$ is the mean-square dipole moment of the chains of $x$ repeating units. In fact, the theoretical values of this quantity for $\mathrm{PMCI}^{14}$ and $\mathrm{PDCI}^{13}$ are 2.34 and $2.48 \mathrm{D}$, respectively, in reasonable good agreement with the experimental results, 2.39 and $2.62 \mathrm{D}$. In the glassy state, generalized microbrownian motions occurring in the liquid state become frozen and the dielectric activity detected in this state mainly arises from conformational transitions taking place by rotations about the angles $\left(\chi_{1}, \chi_{2}\right.$, and $\left.\chi_{3}\right)$ indicated in Fig. 1.

Conformational transitions taking place either in the side groups alone or coupled with local motions in the main chain produce considerable mechanical and dielectric activity in these polymers. Thus the intensity of the mechanical $\beta$ absorption of PDCI, expressed in terms of the loss relaxation modulus and shown in Fig. 10, is much higher than that of the glass-rubber relaxation process. ${ }^{13}$ The fact the intensity of this relaxation decreases and becomes broader for $\mathrm{PMCI}^{14}$ suggests that the absorption is mainly produced by motions taking place in the side chains containing cyclohexyl side groups. Both the mechanical and dielectric $\beta$ relaxation of 


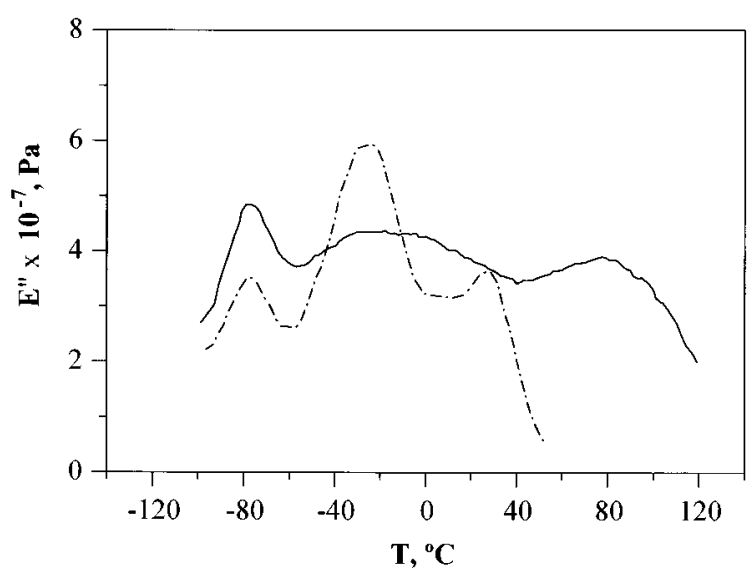

Figure 10. Mechanical relaxation spectra, taken from Refs. 13 and 14, for PDCI (continuous curve) and PMCI (discontinuous curve).

both PMCI and PDCI extends over the same interval of temperature, thus suggesting that similar molecular mechanisms may produce both relaxation processes.

The ac dielectric isochrones for PDCI obtained at frequencies above $1 \mathrm{~Hz}$ present a single $\beta$ peak. ${ }^{13}$ Similarly, a relatively sharp peak exhibits the global depolarization curves plotted against temperature. However, when low frequency isochrones are obtained from partially depolarized curves by means of Eq. (6), one can see that the $\beta$ peak in the isochrones below $10^{-4} \mathrm{~Hz}$ resolves into two well-defined peaks each of which is presumably associated with molecular motions of a determined side group. These two peaks severely overlap at frequencies above $10^{-4} \mathrm{~Hz}$.

Both the mechanical and the dielectric $\beta$ relaxation of PMCI exhibit a broad and complex pattern. For example, the mechanical $\beta$ absorption of PMCI, unlike that of PDCI, is broad and its intensity is similar to that of the glass-rubber relaxation (Fig. 10). ${ }^{14}$ The global TSDC curves also exhibit a broad peak whose intensity is nearly one half of that of PDCI. However, the ac isochrones obtained for PMCI from the partial TSDC curves present a $\beta$ absorption which seems to be the result of three overlapping peaks. The complexity of this peak reflects a presumable copolymeric nature of the PMCI chains. Actually, monoesterfication of the itaconic acid $(\mathrm{HOOC}-) \mathrm{C}\left(-\mathrm{CH}_{2} \mathrm{COOH}\right)=\mathrm{CH}_{2}$ with cyclohexylmethanol may occur in the two acid residues, giving rise to two monomers: (HOOC-) $\mathrm{C}\left(-\mathrm{CH}_{2} \mathrm{COOCH}_{2} \mathrm{C}_{6} \mathrm{H}_{11}\right)=\mathrm{CH}_{2}$ and $\left(\mathrm{C}_{6} \mathrm{H}_{11} \mathrm{CH}_{2} \mathrm{OOC}-\right) \mathrm{C}\left(-\mathrm{CH}_{2}-\mathrm{COOH}\right)=\mathrm{CH}_{2}$. Though it is expected that the fraction of the former monomer may be lower than that of the latter, its presence in the mixture may still be significative and the chains will be copolymers rather than homopolymers. Consequently, $\left(\mathrm{C}_{6} \mathrm{H}_{11} \mathrm{CH}_{2} \mathrm{OOC}-\right) \mathrm{C}\left(-\mathrm{CH}_{2} \mathrm{COOH}\right)-\mathrm{CH}_{2}$ and $(\mathrm{HOOC}-) \mathrm{C}\left(-\mathrm{CH}_{2}-\mathrm{COOCH}_{2} \mathrm{C}_{6} \mathrm{H}_{11}\right)-\mathrm{CH}_{2}$ structures coexist along the chains producing the complicated pattern observed in the spectra.

The relaxation spectra of acrylic and methacrylic polymers esterified with cyclohexylmethanol present a prominent $\beta$ absorption, centered at $-80{ }^{\circ} \mathrm{C}$ at $1 \mathrm{~Hz}$. The apparent universality of this relaxation in this and other polymers containing cyclohexyl side groups in their structure led
Heijboer $^{8}$ to suggest that the relaxation could be produced by chair-to-inverse chair conformational transitions of the cyclohexane ring. It was further postulated that the isochrones of polymers containing cyclohexyl groups in their structure would present the same relaxation. ${ }^{9}$ However, a transition of this kind would not produce dielectric activity by itself, but at least it is coupled with molecular motions of other bonds of either the side groups or the main chain. Recent molecular dynamics calculations $^{22}$ suggest that substitutions in the cyclohexane ring mainly occur in equatorial position, the substitution in axial position being lower than $2 \%$. Similar conclusions were reached from the analysis of the NMR spectra of molecular compounds containing monosubstituted and disubstituted cyclohexane rings in their structure. ${ }^{23}$ Since the population of the states of lowest energy increases as the temperature goes down, substantial conformational transitions in the cyclohexane ring are not expected at temperatures well below the glass transition temperature.

In spite of the fact that both PDCI and PMCI contain cyclohexyl groups in their structure, the mechanical and the dielectric relaxation spectra of these polymers do not exhibit either mechanical or dielectric significant activity centered in the vicinity of $-80{ }^{\circ} \mathrm{C}$ at $1 \mathrm{~Hz}$ where poly(cyclohexyl acrylate) and poly(cyclohexyl methacrylate $)^{8}$ present high intensity absorptions. In this region of the spectra, only a weak dielectric activity is detected for the itaconate polymers used in this study. It can be concluded, however, that the strong $\beta$ absorption exhibited by the mechanical, dielectric, and TSDC spectra of both PDCI and PMCI chains is presumably produced by molecular motions of the side groups.

The low intensity of the mechanical $\alpha$ relaxation in comparison to that of the $\beta$ process in itaconate polymers suggests that the mechanical activity in the $\beta$ region mainly arises in these polymers from molecular motions in the side groups whose complexity increases with temperature. However, the mechanical activity in the glass-rubber relaxation mainly comes from molecular motions in the skeletal bonds. These motions are severely restricted by the side groups and hence the comparatively low intensity of this latter absorption.

On the contrary, the higher intensity of the dielectric $\alpha$ glass-rubber relaxation in comparison with that of the $\beta$ process presumably arises from the fact that the limited microbrownian motions of the main-chain taking place in the glass-rubber transition still facilitate the orientation of the dipolar entities in the electric field and hence the high intensity of the glass-rubber relaxation process.

Finally, a few comments on the small changes observed for the heat capacity of polyitaconates in the glass transition, to which we have referred in the section on the experimental characterization of these polymers. As is well known, when an uncrystallizable liquid passes during cooling through the liquid $\rightarrow$ glass transition, a rapid decrease of heat capacity from liquid-like to crystal-like values occurs. This is a consequence of the fact that the liquid degrees of freedom become kinetically inaccessible. The calorimetric pattern in the glass transition can be used to define two kinds of liquids: fragile liquids and strong liquids. Fragile liquids are characterized by their capability to reorganize to structures that 
fluctuate over a wide variety of particles or bond group orientations, in the vicinity of the glass transition temperature. On the contrary, the resistance to structural changes is the principal characteristic of strong liquids. ${ }^{24}$ Consequently, fragile liquids exhibit big jumps in $\Delta C_{p}$ at $T_{g}$, while the jumps are very small in strong liquids. Polymers are the systems which show the most fragile liquid character, whereas silicon oxide, germanium oxide, inorganic salts, etc., are representative of strong systems. Therefore the low changes observed in $\Delta c_{p}$ at $T_{g}$ in polyitaconates suggest a strong liquid character for these substances. Consequently, the thermal behavior of these polymers in the vicinity of the glass transition temperature also reflects the limited conformational space of the backbone of polyitaconate chains.

${ }^{1}$ N. G. McCrum, B. E. Read, and G. Williams, Anelastic and Dielectric Effects in Polymeric Solids (Wiley, New York, 1967).

${ }^{2}$ M. Doi and S. F. Edwards, The Theory of Polymer Dynamics (Clarendon, Oxford, 1986).

${ }^{3}$ W. H. Stockmayer, Pure Appl. Chem. 15, 539 (1967).

${ }^{4}$ K. Adachi and T. Kotaka, Macromolecules 17, 120 (1984).

${ }^{5}$ E. Riande and E. Saiz, Dipole Moments and Birefringence of Polymers (Prentice-Hall, Englewood Cliffs, NJ, 1992).
${ }^{6}$ J. Johari, Ann. (N.Y.) Acad. Sci. 279, 117 (1976).

${ }^{7}$ G. Williams, Adv. Polym. Sci. 33, 59 (1979).

${ }^{8}$ J. Heijboer, Ph.D. thesis, Leiden University, Leiden, The Netherlands, 1972.

${ }^{9}$ J. Heijboer, Ann. (N.Y.) Acad. Sci. 279, 105 (1976).

${ }^{10}$ G. D. Smith and R. H. Boyd, Macromolecules 24, 2731 (1991).

${ }^{11}$ E. Helfand, Science 226, 647 (1984).

${ }^{12}$ M. D. Ediger and D. B. Adolf, Adv. Polym. Sci. 116, 73 (1994).

${ }^{13}$ R. Díaz-Calleja, E. Saiz, E. Riande, L. Gargallo, and D. Radic, Macromolecules 26, 3795 (1993).

${ }^{14}$ R. Díaz-Calleja, E. Saiz, E. Riande, L. Gargallo, and D. Radic, J. Polym. Sci. Part B: Polym. Phys. 32, 1069 (1994).

${ }^{15}$ M. Yazdani-Pedram, L. Gargallo, and D. Radic, Eur. Polym. J. 21, 707 (1985).

${ }^{16}$ R. Fuoss and J. G. Kirkwood, J. Am. Ceram. Soc. 63, 385 (1941).

${ }^{17}$ J. van Turnhout, Thermally Stimulated Discharge of Polymer Electrets (Elsevier, Amsterdam, 1975).

${ }^{18} \mathrm{~J}$. Vanderschueren and J. Gasiot, in Thermally Stimulated Relaxation in Solids, edited by P. Bräunlich (Springer, Berlin, 1979).

${ }^{19}$ C. Bucci, R. Fieschi, and G. Guidi, Phys. Rev. 148, 816 (1966).

${ }^{20}$ H. Shimizu and K. Nakayama, J. Appl. Phys. 74, 1597 (1993).

${ }^{21}$ M. M. Perlman and S. Unger, J. Appl. Phys. 45, 2389 (1974).

${ }^{22}$ E. Saiz and E. Riande, J. Chem. Phys. 103, 3832 (1995).

${ }^{23}$ R. Díaz-Calleja, E. Riande, J. San Roma' n, and V. Compan , Macromole cules 27, 2092 (1994).

${ }^{24}$ C. A. Angell, Science 267, 1924 (1995). 